\title{
Multipath Video Streaming Using GRA Network Ordering Algorithm without Rank Inconsistency
}

\author{
Árpád Huszák, Sándor Imre \\ Budapest University of Technology and Economics, \\ Department of Telecommunications, Budapest, Hungary \\ Email: \{huszak,imre\}@hit.bme.hu
}

\begin{abstract}
Simultaneous connection to several networks through multiple interfaces is possible with today's mobile terminals. In order to efficiently utilize the interfaces' capabilities and increase the quality of the streamed video, multipath streaming can be used. Resource intensive applications can deliver high bitrate streams over multiple paths by cumulating the available bandwidth of the different subpaths. In this paper we propose a multipath streaming method that chooses a set of paths maximizing the overall quality at the client. While the available paths have different bandwidth, delay and loss probability constrains, the packet distributor must take the video packet importance and the dependencies between packets into account. In order to efficiently distribute the packets, the link must be ordered based on the network attributes. Grey Relational Analysis (GRA) is a promising algorithmic approach that can realize dynamic interface ordering with multiple alternatives (interfaces) and attributes (network parameters). However similarly to some other decision methods, GRA also suffers from rank reversal phenomenon. Transmitting the reference video frames on the most reliable links will decrease the loss probability of important data packets and increase the measured video quality. The change of link order can lead to frequent handovers causing the degradation of the observed video quality.
\end{abstract}

Keywords: Multipath streaming, QoS, Grey Relational Analysis, rank reversal

\section{INTRODUCTION}

The popularity of multimedia applications is increasing on the Internet in both wired and wireless environment. Despite the development of new wireless access technologies that provide higher bandwidth to the users, multimedia streaming applications still suffer from limited and highly varying bandwidth. Although the offered bandwidth is getting higher, high quality video streams usually can not be transmitted on a single link. In order to utilize the advantages of the heterogeneous network model, mobile devices are now being built as multihomed multi-functioning wireless terminals. Multipath video streaming has recently been proposed as a solution to overcome the strict mobile network limitations. In order to increase the quality of streams using multihomed devices that have multiple network interfaces independently connected to different networks, the available interfaces must be effectively utilized. Higher bandwidth can be provided to the users if joint links are used. The independent but joint links can be successfully utilized by a streaming application in order to achieve high quality streaming. In this paper an interface selection algorithm for multipath MPEG video streaming is presented. The missing packets in a video frame not only lead to reduced quality of the frame but also result in the propagation of distortion to successive frames. This is due to inter-frame-video compression algorithms such as MPEG, which exploit temporal correlation between frames to achieve higher compression. The proposed scheme takes the video packet importance and the dependencies between packets into account 
and transmits the reference video frames on the most reliable links. Using the proposed packet distributor the loss probability of important data packets can be decreased, hereby increasing the measured video quality.

In our video multipath streaming proposal the available links must be ordered to determinate the best interfaces for the most important packets. The simplest ordering technique is to make the network ordering based on one network parameter, e.g. packet loss, however it is not the best solution. The network selection algorithm must depend on the requested services and other network parameters, such as throughput, delay, jitter, cost, signal strength etc. The ordering algorithm must be able to determine automatically the best interface and the interface order that fulfills the user's requirements.

Multi Attribute Decision Making (MADM) methods can be utilized for network ordering purposes. MADM algorithms are used for determining the ranking of alternatives in terms of their desirability with respect to multiple criteria that can influence the decision. The main goals of these solutions are to avoid frequent handover processes and offer acceptable QoS characteristics for different types of applications. In the recent years a new network selection method has been studied based on Grey System Theory [1]. The theory has been proven to be useful for dealing with poor, incomplete, and uncertain information. Grey Relational Analysis (GRA) is part of Grey System Theory, which is suitable for decision making with complicated interrelationships between multiple factors and variables. GRA has been successfully applied in network selection process as well [2]-[5]. Similarly to other MADM techniques, GRA has been also criticized for its possible rank reversal phenomenon, which means that the relative rankings of two decision alternatives could be reversed when a decision alternative is added or deleted. Rank reversal is a disadvantageous behavior even for our proposed content-aware multipath video streaming method. If not all the available links are used for the multipath transmission, the network selection method, which automatically switches to the best network, may cause frequent handovers due to rank inconsistency. Each appearance of a new network or a link disappearance may trigger a handover process, increasing delay, causing gap in the transmission or even disconnecting the terminals.

In this paper we introduce a content-aware and multipath video streaming technique, which uses a modified GRA to make the ordering of links. We made innovation in both multipath streaming and GRA procedure. The main difference of our contentaware and multipath streaming technique from the other multipath streaming solutions is that we use a sender side buffer to heap up more data in order to utilize the available links more efficiently.

We have analyzed the proposed multipath streaming scheme both analytically and with simulations to estimate the quality improvement of the transmitted video streams. Regarding the GRA procedure used for network ordering, we propose a solution to eliminate the ordering inconsistency of GRA-based network selection algorithm. In order to avoid the effects of the rank reversal, we have analyzed the GRA-based network selection algorithm to find the reason of the phenomenon. We have found that modifying the normalization method, the probability of rank reversal can be significantly decreased or even eliminated. In order to justify our theoretical assumptions, we have examined the efficiency of the proposed solutions.

The rest of this paper is organized as follows. Review of related works in multipath video streaming and network selection decision methods, especially GRA technique is presented in Section II. In Section III we introduce our content-aware multipath streaming solution with sender side heap up buffer. Section IV describes our solutions for reducing and eliminating the rank reversal phenomenon for GRAbased network selection. The obtained performance results are presented in Section V. Finally, we 
summarize our paper and make the conclusions in the last section.

\section{BACKGROUND AND RELATED WORKS}

Our work combines the multipath video streaming and network ordering procedure, therefore in this section the previous studies of both techniques are introduced.

\section{A. Multipath Video Streaming}

Multipath multimedia streaming has become an attractive solution due to its increased throughput and adaptive load balancing [6]. An overview of the benefits and use of path diversity for media streaming is introduced in [7][8]. In these works different approaches for media coding and streaming over multiple paths are examined, together with architectures for achieving path diversity between single or multiple senders and a single receiver. Novel solutions were also presented to improve the quality of streaming applications. The authors of [9][10] study video streaming using feedback-based reference picture selection schemes and path diversity. In [11] multipath streaming methods are examined from the application transparency point of view. A distributed sender-driven streaming solution is presented in [12], where servers collaboratively adapt to the network status in order to provide the media client with a superior video quality. Most of the related works analyze the multipath streaming solution using simulations; however the authors of [13] deployed a testbed for the measurements. The multipath streaming was also investigated from congestion point of view in [14][15]. In the latter one, a fast heuristicbased algorithm is presented, that is built on loadbalancing principles.

Multihomed protocols such as SCTP [16] provide the possibility of multiple link connections. However, the current protocol version does not allow to use multiple path simultaneously. Ye et al. [17] introduced an independent per path congestion control principle for standard SCTP, while in [18] a similar solution is presented with load sharing techniques.

\section{B. Network Ordering}

Neither of the previously developed multipath streaming schemes deal with ranking of links, however it plays an important role in each proposal. In our solution GRA (Grey Relational Analysis) was used for this purpose, which is one of the Multi Attribute Decision Making (MADM) techniques.

Numerous network selection methods were studied in the past decade. All these decision algorithms are used for determining the ranking of competitive network access possibilities and making the best choice for the user. In the traditional methods such as [19]-[22], only the radio signal strength (RSS) threshold and hysteresis values are considered. The simple RSS-based decision is not sufficient because it does not take into account the provided services and features of different access technologies.

Making the decision based on one parameter is usually inadequate, therefore MADM algorithms such as SAW, TOPSIS, AHP (Analytic Hierarchy Process), ELECTRE, etc. have been developed and described in [22]-[29]. These solutions determinate the preferred network using technology specific features such as bandwidth, delay, packet loss probability, cost of network usage, etc. Roveri et al. [30] used weight factors to reflect the dominances of the particular requirements with respect to the user. GRA-based network selection method [2]-[5] is an alternative technique for determination of the network priority order. Similarly to TOPSIS and some ELECTRE variants, GRA also suffers from rank inconsistency.

A robust MADM algorithm must ensure that the order of alternatives does not change when an alternative, which is not the best, is removed or replaced by another alternative. Therefore, if an algorithm suffers from the ranking abnormality problem, the ranking order is not stable.

\section{1) Grey Relational Analysis (GRA)}

Grey System Theory was introduced in [1] to analyze the relational grade for several discrete sequences and select the best sequence. One of the 
sequences is defined as reference sequence presenting the idea situation. The grey relationship between the reference sequence and the other sequences can be determined by calculating the Grey Relational Coefficient (GRC) according to the level of similarity and variability. The technique is appropriate and effective for network selection purposes as well. The GRA-based network selection method can be implemented following the steps bellow:

1. Classifying the network parameters (lower-thebetter, higher-the-better)

2. Defining the upper and lower bounds of the parameters

3. Normalizing the parameters

4. Calculating the Grey Relational Coefficient (GRC)

5. Ranking the networks according to the GRC values

To calculate the GRC, the network parameters must be categorized first. For delay, cost, etc. parameters the smaller-the-better class is used, while other ones like throughput and signal strength parameters belong to the larger-the-better category. Before calculating the GRC for each parameter of the network, the data needs to be normalized to eliminate dimensional units. Assuming that $n$ possible networks $\left(S_{1}, S_{2}, \ldots, S_{n}\right)$ are compared, and each network has $k$ parameters, the upper bound $\left(u_{j}\right)$ is defined as $\max \left\{s_{1}(j), s_{2}(j), \ldots\right.$, $\left.s_{n}(j)\right\}$, and the lower bound $(l j)$ as $\min \left\{s_{l}(j), s_{2}(j), \ldots\right.$, $\left.s_{n}(j)\right\}$, where $j=1,2, \ldots, k$. In case of smaller-thebetter attribute, the normalized value of $s_{i}(j)$ parameter can be calculated as follows:

$$
s_{i}^{*}(j)=\frac{u_{j}-s_{i}(j)}{u_{j}-l_{j}}
$$

Similarly, the normalized value of a larger-thebetter parameter:

$$
s_{i}^{*}(j)=\frac{s_{i}(j)-l_{j}}{u_{j}-l_{j}}
$$

The attributes of a network can be represented as a row matrix, where the elements of the matrix are the normalized values of $k$ different network attributes.

$$
S=\left[\begin{array}{lllll}
s^{*}(1) & s^{*}(2) & s^{*}(3) & \ldots & s^{*}(k)
\end{array}\right]
$$

While $s_{i}{ }^{*}(j)$ parameters are maximized in 1 , the most preferable network can be always described as $s_{i}{ }^{*}(j)=1$, where $j=1,2, \ldots, k$ and $k$ is the number of network parameters used for the decision. Utilizing this behavior of the normalizing algorithm, the ideal network is considered as $S=\left[\begin{array}{llll}1 & 1 & \ldots & 1\end{array}\right]$.

If there are $N$ competing networks to choose from, the previous row matrix (3) can be extended to an $N \times k$ matrix, which contains all the parameters that play role in the network selection procedure. The matrix can be determined as follows:

$$
S_{N}=\left[\begin{array}{lllll}
s_{1}^{*}(1) & s_{1}^{*}(2) & s_{1}^{*}(3) & \ldots & s_{1}^{*}(k) \\
s_{2}^{*}(1) & s_{2}^{*}(2) & s_{2}^{*}(3) & \ldots & s_{2}^{*}(k) \\
\ldots & & & & \\
s_{N}^{*}(1) & s_{N}^{*}(2) & s_{N}^{*}(3) & \ldots & s_{N}^{*}(k)
\end{array}\right]
$$

The final step of the GRA-based network selection algorithm is to calculate the Grey Relational Coefficient (GRC). The value of the GRC parameter is calculated by the following equation, where $w_{j}$ is the weight of each parameter and $i(1 \leq i \leq N)$ is the network index:

$$
G R C_{i}=\frac{1}{\sum_{j=1}^{k} w_{j}\left|s_{i}^{*}(j)-1\right|+1}
$$

The network with the largest GRC is the most desirable one.

\section{2) Rank Reversal Phenomenon in MADM algorithms}

Most of the MADM-based ranking algorithms use normalization and upper/lower bounds determinations of the network parameters. Rank reversals in the SAW, TOPSIS, AHP, GRA methods are caused by the changes of normalized attribute values. Some proposals [27],[31] were presented to avoid rank reversal in AHP and TOPSIS, while Barzilai and Golany [32] have proved that no normalization can prevent rank reversal. However, normalization is often 
necessary for most of the MADM approaches to eliminate different dimensional units. GRA also suffers from the rank reversal as the following example shows. The attributes and its normalized values used for the network selection decision are shown in Table I.

\begin{tabular}{|c|c|c|c|c|c|c|c|c|c|c|c|}
\hline \multicolumn{2}{|c|}{ Network } & \multicolumn{2}{|c|}{ Delay } & \multicolumn{2}{|c|}{ Jitter } & \multicolumn{2}{|c|}{ Loss rate } & \multicolumn{2}{|c|}{ Throughput } & \multicolumn{2}{|c|}{ Cost } \\
\hline & GRC & $\mathrm{ms}$ & norm & $\mathrm{ms}$ & norm & ratio & norm & Mbps & norm & $\$ / M B$ & norm \\
\hline$\# 1$ & 0.339 & 10 & & & & 0.112 & & & 0.79 & & 0.978 \\
\hline$\# 2$ & 304 & 200 & & 10 & 0.916 & 0.01 & & 80 & 0.885 & 11 & \\
\hline \#3 & 0.343 & 100 & 0.526 & 21 & 0.458 & 0.1 & 0.107 & 90 & 1 & 0.8 & 1 \\
\hline \#4 & 0.281 & 50 & 0.789 & 32 & 0 & 0.0003 & & & 0.01 & 4 & 0.652 \\
\hline$\# 5$ & 0.302 & 160 & 0.21 & 12 & 0.833 & 0.012 & 0.916 & & 0 & 3 & 0.76 \\
\hline
\end{tabular}

\section{TABLE I: GRA-BASED NETWORK SELECTION DECISION}

According to the normalization equations (1) and (2) of the GRA-based decision making, we can find 0 and 1 normalized values for each network attribute. Normalized value 1 means that the network offers the best performance from the given parameter point of view, while 0 means the opposite. By calculating the GRC (5) the ranking order can be determined. In the example presented in Table I, network \#3 is the best choice and \#2 is the worst one, if the weights of the attributes are considered equal.

Supposing that the worst network (\#2) is not reachable any more, the normalized values and calculated GRCs will be changed as shown in Table II.

\begin{tabular}{|c|c|c|c|c|c|c|c|c|c|c|c|c|}
\hline \multicolumn{2}{|c|}{ Network } & \multicolumn{2}{|c|}{ Delay } & \multicolumn{2}{|c|}{ Jitter } & \multicolumn{2}{c|}{ Loss rate } & \multicolumn{2}{c|}{ Throughput } & \multicolumn{2}{c|}{ Cost } \\
\hline & GRC & Ms & norm & ms & norm & ratio & Norm & Mbps & norm & \$MB & norm \\
\hline \hline$\# 1$ & $\mathbf{0 . 3 3 5}$ & 10 & 1 & 8 & 1 & 0.112 & 0 & 10 & 0.079 & 1 & 0.937 \\
\hline$\# 2$ & & -- & -- & -- & -- & -- & -- & -- & -- & -- & -- \\
\hline$\# 3$ & 0.329 & 100 & 0.4 & 21 & 0.458 & 0.1 & 0.107 & 90 & 1 & 0.8 & 1 \\
\hline$\# 4$ & 0.234 & 50 & 0.733 & 32 & 0 & 0.0003 & 1 & 4 & 0.01 & 4 & 0 \\
\hline$\# 5$ & 0.252 & 160 & 0 & 12 & 0.833 & 0.012 & 0.891 & 3 & 0 & 3 & 0.312 \\
\hline
\end{tabular}

\section{TABLE II: GRA-BASED NETWORK SELECTION DECISION WITHOUT NETWORK \#2}

After the removal of network \#2 the best network was changed to network \#1; however the network parameters were not changed.

III. CONTENT - AWARE MULTIPATH STREAMING
We consider a client-server network topology, where the client can access the server simultaneously through different paths. The independent paths are characterized with different bandwidth $\left(b w_{i}\right)$, packet loss ratio $\left(p_{i}\right)$, network delay $\left(d_{i}\right)$, etc. parameters belonging to path $i$.

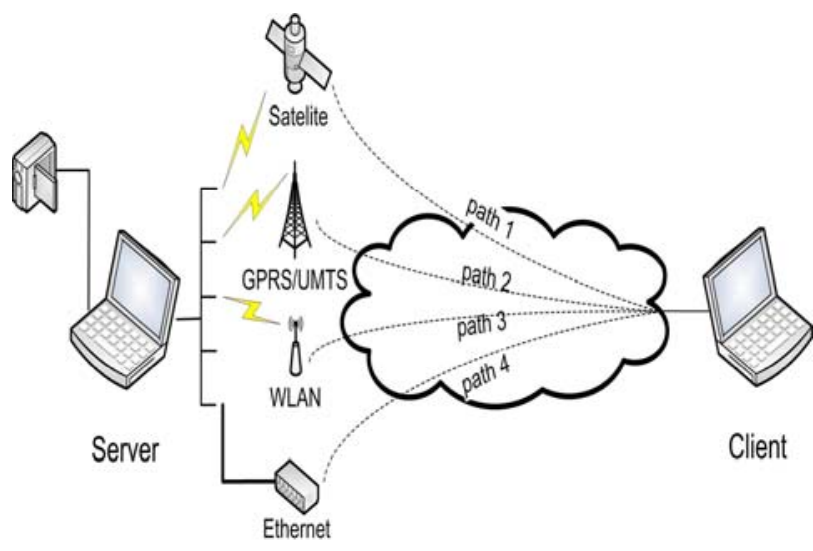

Figure 1: Multipath data delivery

Network delay parameter plays role when a strict playout buffer is used at the receiver. If the transmitted packet is received after the playout time, it can be considered as lost packet, therefore we do not deal with the delay parameter separately. We consider that total packet loss probability $\left(\pi_{i}\right)$ on path $i$ contains the loss events due to channel errors and late reception of video packets:

$$
\pi_{i}=p_{i}+\operatorname{Pr}\left(d_{i}>d_{p b u f f}\right),
$$

where $d_{p b u f f}$ is the playout buffer delay. The overall packet loss ratio for the multipath transmission is the weighted average of the total packet loss probabilities

$$
\pi=\frac{d_{1}}{d} \pi_{1}+\frac{d_{2}}{d} \pi_{2}+\ldots+\frac{d_{n}}{d} \pi_{n}=\sum_{i=1}^{n} \frac{d_{i}}{d} \pi_{i}
$$

where $d_{i}$ is the data amount transmitted on the $i^{\text {th }}$ link and $d$ is the total size of the delivered video stream.

The transmitted video quality can be increased if the total packet loss probability is minimized; therefore the available paths are used in order of the links quality $\left(\pi_{i}\right)$. The lowest packet loss ratio paths 
must be utilized first and the worst channels must be used only if necessary. The impact of packet loss ratio is the most significant, however other network attributes must be also considered for the network order determination. For example, the physical parameters of a link can be very good, but if the [\$/byte] cost of usage is extremely high, the link will not be chosen. The cost has no impact on the video quality, but it can be an attribute of the network order decision algorithm.

Further video quality improvement can be achieved if the packet contain is also taken into account. The MPEG coded video streams use different frame types (I-, P- and B-frames) with inter-frame dependencies. We propose a contentaware packet distributor scheme in order to minimize the distortion propagation in the stream by forwarding the I-frames on the most reliable links. In our proposed scheme a buffer is applied that heaps up $B_{f}$ number of frames causing $d_{B}$ extra delay. In every $d_{B}$ time we distribute the stored data packets between the interfaces and the heap up procedure starts again. This method makes it possible to efficiently distribute the data packets of the video stream. If longer delay $\left(d_{B}\right)$ is used, more I-frames can be forwarded to the most reliable link, but the overall delay $(D)$ will be also higher.

$$
D=d_{B}+\max \left(d_{i}\right)
$$

The acceptable delay threshold depends on the used multimedia application. In case of one-way live media applications it can be even few seconds, but if two-way interactive application is used the perceptual tolerance of the user is around 150 $200 \mathrm{~ms}$. The limitations of two-way interactive applications are too strict for our proposed interface selection algorithm, but for pre-recorded and oneway live video streams this solution is acceptable from the delay point of view.

The streaming server is connected to the channels through buffer interfaces, which are modeled as FIFO queues. These queues are refilled at every $d_{B}$ time from the heap up buffer. The interface buffers' sizes are determined by the paths bandwidth and the heap up buffer delay $\left(d_{B}\right)$. The interface buffers must be purged in $d_{B}$ time to be able to receive the next amount of data. The server side interface selection scheme is illustrated in 0

The video quality improvement is gained due to the efficient packet distribution from the heap up buffer to the interface buffers. Due to the proposed content-aware interface selection method the distribution of data belonging to I-, P-, B-frames on the independent links will be totally different from the original video frame structure. On the reliable channels more I-frame data will be delivered and less B-frames, while on links with high loss probability mostly B-frames will be transmitted.

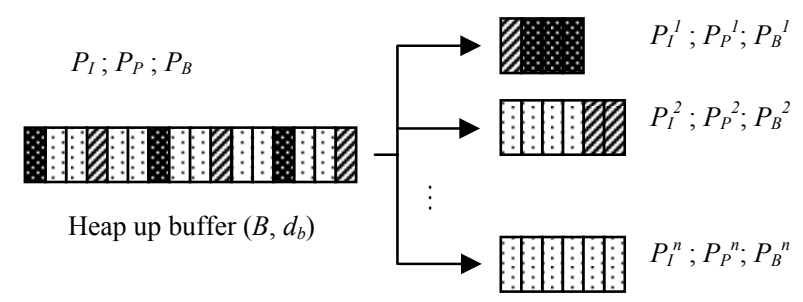

Figure 2: Frame distribution on the interfaces

The possibility of the data bytes belonging to I-, $\mathrm{P}-$ and $\mathrm{B}-$ frames of the video stream is consequently $P_{I}, P_{P}$ and $P_{B}$. If the heap up delay $\left(d_{B}\right)$ is long enough, these possibilities are similar in the heap up buffer. Due to the proposed interface selection algorithm the distribution of $\mathrm{I}-, \mathrm{P}-, \mathrm{B}-$ frame data bytes in the interface buffers will be different from the original video frame data distribution.

The possibility that a data byte belongs to an Iframe in the most reliable interface's buffer can be calculated as follows:

$$
P_{I}^{1}=\min \left(\frac{P_{I} B}{b_{1}}, 1\right)
$$

Parameter $B$ is the size of data heaped up, while $B_{1}$ is the buffer size of the first (most reliable) interface. We use the $\min ()$ function to keep the probability variable bellow or equal to 1 . 
In order to analyze the behavior of the proposed scheme we can calculate the distribution of $\mathrm{I}_{-}, \mathrm{P}-$, B-frame data bytes in the interface buffers $\left(P_{I}^{i}, P_{P}{ }^{i}\right.$ and $\left.P_{B}{ }^{i}\right)$. The $\mathrm{I}-$ frame data probability in the $i^{\text {th }}$ interface buffer is:

$$
P_{I}^{i}=\max \left(0, \min \left(\frac{P_{I} B-\sum_{n=1}^{i-1} b_{n}}{b_{n}}, 1\right)\right)
$$

Similarly, the $\mathrm{P}-$ and $\mathrm{B}-$ frame data ratios in the $i^{\text {th }}$ interface buffer can be calculated as follows:

$$
\begin{gathered}
P_{P}^{i}=\max \left(0, \min \left(\left(\max \left(0, \min \left(\frac{P_{P} B-\sum_{n=1}^{i} P_{P}^{n} b_{n}}{b_{n}}, 1\right)\right)-P_{I}^{i}\right), 1\right)\right) \\
P_{B}^{i}=\max \left(0, \min \left(\left(\max \left(0, \min \left(\frac{P_{B} B-\sum_{n=1}^{i} P_{B}^{n} b_{n}}{b_{n}}, 1\right)\right)-P_{I}^{i}-P_{P}^{i}\right), 1\right)\right)
\end{gathered}
$$

The video quality depends on the number of lost packets and on the content of the missing packet. The loss of a B-frame packet will not reduce the quality so significantly than an I-frame loss, due to frame dependencies of the MPEG video stream structure. The aim of the proposed scheme is to reduce the $\mathrm{I}-$ and $\mathrm{P}-$ frame data losses.

The number of lost I-, P- and B-frame packets can be estimated based on the link packet loss probability $\left(\pi_{i}\right)$ and the previously introduced frame ratio ratios on the links, see equations (10)-(12). Considering that the interfaces are ordered according to the links' packet loss ratio $\left(\pi_{1} \leq \pi_{2} \leq \ldots \leq \pi_{n}\right)$ the number of lost packets, which contain I-frame data, can be calculated as follows:

$$
N_{I}=\sum_{i=1}^{n} \frac{P_{I}^{i} b_{i}}{S} \pi_{i}
$$

The packet size is signed with $S$, while $b_{i}$ stands for the buffer size of the $i^{\text {th }}$ interface. Similarly to (13), the number of $\mathrm{P}-$ and $\mathrm{B}-$ frame packet losses can be also estimated.

In this section the effect of packet loss ratios was studied as the primary network parameter. The packet probability and the delay have the most significant impact on the video quality, where the late packets are handled as lost packets. In our investigations the packet losses due to late delivery were not distinguished, therefore we did not need to separately handle the delay parameter.

Besides the loss ratio, numerous other network parameters can be used to determinate the interface order. Some of these parameters, such as cost, link availability ratio, etc. have no immediate impact on the video quality, however the types of these parameters must be also taken into consideration.

\section{ALTERNATIVE NORMALIZATION METHODS FOR GRA-BASED NETWORK SELECTION}

GRA is an effective tool for network ordering purposes. Similarly to other solutions GRA also suffers from rank reversal phenomenon. It can be easily proved that the reason of this observable fact is the normalization method. The result of the Grey Relational Coefficient (GRC) equation (5) will change only if the normalized network attributes also change, however we assume that the network parameters are constant.

Although the network parameters, such as bandwidth, delay, loss rate, etc. are considered constant, the normalized value of these attributes can be changed if one of the networks is not reachable any more. This can happen if one of the disappearing networks attributes has the highest or lowest value. In this case the attribute order will not change of course, but the distance between the normalized values may vary significantly as the example below shows.

As presented in Figure 3, the normalized values calculated with equation (1) and (2) significantly differ, when the highest element was removed. While the difference between the two normalized values in the middle is only $0.18-0.15=0.03$, after the removal of the highest attribute, the difference becomes 0.72 $0.6=0.12$. Although the real data was not changed, the significant variance of the normalized values can modify the rank order of the networks. 


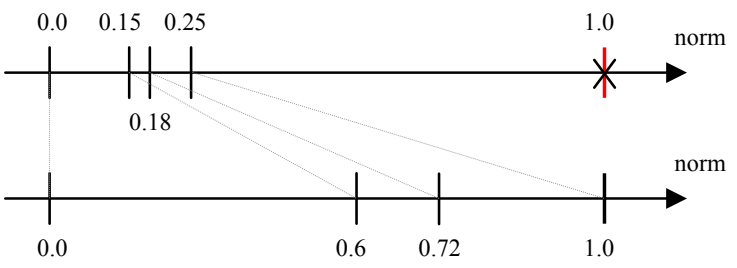

Figure 3: The effect of removing the highest value on the normalized value

Our main goal was to reduce the effect of the normalization on the rank order. We propose three different methods to replace the actual normalization procedure described by equations (1) and (2).

Our first normalization solution keeps the normalized values unchanged by determine absolute min-max values of an attribute. By keeping the normalized values unchanged the calculated GRC will be the same even if a network is not reachable anymore, therefore the rank reversal phenomenon is eliminated. The modified normalized value of a smaller-the-better attribute can be calculated as follows:

$$
s_{i}^{*}(j)=\frac{E_{\max _{j}}-s_{i}(j)}{E_{\max _{j}}-E_{\min j}}
$$

Similarly, the normalized value of a larger-thebetter parameter:

$$
s_{i}^{*}(j)=\frac{s_{i}(j)-E_{\min _{j}}}{E_{\max _{j}}-E_{\min _{j}}}
$$

The variable $E_{\min j}$ and $E_{\max j}$ stand for absolute minimum and maximum of the $j^{\text {th }}$ network parameter, respectively. The $j^{\text {th }}$ parameter value therefore must be always between $E_{\min } j$ and $E_{\max } j$. For absolute minimum, $E_{\min j}=0$ can be an adequate option, while for absolute maximum $\left(E_{\max }\right)$, the given network attribute behavior must be analyzed.

The second modified normalization method is similar to the previous one, but it uses absolute bound only in the unwanted direction of an attribute. In case of smaller-the-better attribute an absolute maximum is determined, while for larger-the-better parameter an absolute minimum is used. The reason of this solution is that usually those networks are becoming unreachable whose attributes are bad. For example, in case of larger-the-better attribute (e.g., signal strength, throughput) that network will disappear whose signal strength and/or throughput become very low. A network with high signal strength and throughput probably will not become unreachable, therefore only absolute minimum needs to be used in these cases in order to reduce the rank reversal probability.

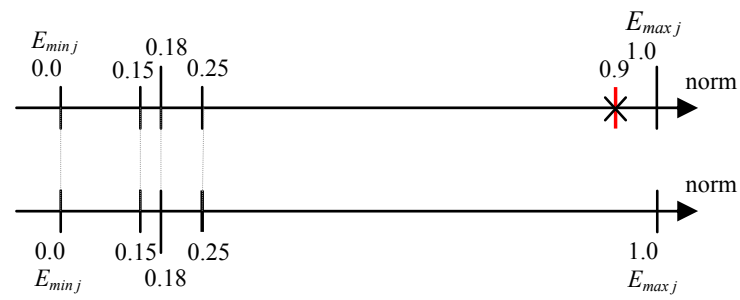

Figure 4: Preventing normalized value changes using absolute min - max attributes

According to the previous assumption, the modified normalized value of a smaller-the-better attribute can be calculated as follows:

$$
s_{i}^{*}(j)=\frac{E_{\max j}-s_{i}(j)}{E_{\max j}-l_{j}}
$$

Similarly, the normalized value of a larger-thebetter parameter is given by:

$$
s_{i}^{*}(j)=\frac{s_{i}(j)-E_{\min j}}{u_{j}-E_{\min j}}
$$

In the above equations $E_{\min j}$ and $E_{\max j}$ are the absolute minimum and maximum of the $j^{\text {th }}$ network parameter that must be determined before the network selection procedure starts. Variable $u_{j}$ is the maximal value of the $j^{\text {th }}$ parameter, and it is defined as $\max \left\{s_{l}(j), s_{2}(j), \ldots, s_{n}(j)\right\}$. The lowest value $(l j)$ is defined as $\min \left\{s_{l}(j), s_{2}(j), \ldots, s_{n}(j)\right\}$.

The secondly introduced normalization technique will not eliminate rank reversal, because the 
normalized value of a parameter can still change if the best network becomes unreachable. We can assume that in most of the cases the best network will not disappear unexpectedly, therefore the normalized values and the calculated GRC probably will not be changed. Using this way of normalization, neither the normalized value of the best attribute will be equal to 1 , nor will the normalized value of the worst attribute be equal to 0 .

In our third normalization solution our aim was to avoid the usage of absolute min-max values. Similarly to the previous solution we proposed that those networks will become unreachable whose parameters are worse. We have constructed the normalization function in such a way that the normalized value of the best parameter be equal to 1 .

The proposed normalization function of a smallerthe-better attribute is as follows:

$$
s_{i}^{*}(j)=\frac{l_{j}}{s_{i}(j)}
$$

The normalized value of a larger-the-better parameter can be calculated similarly:

$$
s_{i}^{*}(j)=\frac{s_{i}(j)}{u_{j}}
$$

In the above equations $u_{j}$ stands for the maximal value of the $j^{\text {th }}$ parameter, which is defined as $\max \left\{s_{l}(j), s_{2}(j), \ldots, s_{n}(j)\right\}$, while $l j$ is the lowest value.

This normalization technique guarantees that the best attribute will be 1 , therefore the distances between the normalized values of an attribute will be higher compared to the secondly introduced normalization technique. The ranking order will be clearer, because the differences of the calculated coefficients (GRC) belonging to different networks will be bigger. The third normalization technique will not solve the rank reversal problem; however it significantly reduces its occurrence probability. To calculate the normalized value of a smaller-the-better attribute (18) we use only the lowest value from the examined parameters $\left(l_{j}\right)$ that is considered as the best choice from the $j^{\text {th }}$ parameter point of view. Therefore the removal of any other network, but not the best one, will not cause the change of the normalized values. The calculation of the normalized values of the larger-the-better parameters (19) is similar, but in these cases the highest value of the $j^{\text {th }}$ parameter $\left(u_{j}\right)$ is used. The normalized values will change only if the network whose $j^{\text {th }}$ parameter equal to $u_{j}$ is removed.

\section{PERFORMANCE EVALUATION}

This section presents and discusses the performance of the proposed algorithms regarding to content-aware packet distribution and modified GRA network ordering technique.

\section{A. Multipath Video Streaming}

This subsection compares our content-aware interface selection solution to continuous and to balanced interface selection methods. The continuous method fills the interface buffers continuously from the video source or the video data storage. This model does not use heap up buffer, therefore the advantage of this method is that no extra delay is introduced. We consider that the links are ordered and the most reliable link buffer is filled first and if the link's capacity is fully utilized, the next interface is selected and so on. The distribution of different frame type data on the links will be similar to the original video frame structure $\left(P_{I}: P_{P}\right.$ : $\left.P_{B}=P_{I}^{i}: P_{P}{ }^{i}: P_{B}{ }^{i}\right)$. Previous work does not apply heap up buffer, hence the actual frame data must be forwarded to the link buffers immediately. The balanced method fills the interface buffers uniformly, using the Round-Robin model. If one of the interface buffers is full, the remaining video data is distributed between the other interfaces, which are not full yet. This Round-Robin based interface selection method does not take the link characteristics into account. The distribution of the video frame type data in the interface buffers will be similar to the original frame structure $\left(P_{I}: P_{P}: P_{B}=\right.$ $\left.P_{I}^{i}: P_{P}{ }^{i}: P_{B}{ }^{i}\right)$. 
We have developed a simulation tool to analyze the behavior of the proposed content-aware interface selection scheme. Each simulation scenario was executed ten times and an average was calculated from the obtained results. The mother and daughter QCIF sequence was used as the reference video. The video sequence was compressed with an MPEG-4 encoder - ffmpeg [35]. The video stream was coded at $25 \mathrm{fps}$ and $300 \mathrm{kbps}$. The frame structure is specified by the $N=10$ and $M=5$ parameters. $N$ and $M$ are the intraframe and interframe coding ratios, which define the sequence of $\mathrm{I}-, \mathrm{P}-$ and $\mathrm{B}$-frames. $N$ specifies the I-frame interval, whereas $M$ determines the I- or P-frame interval. The distribution of the used video data bytes belonging to $\mathrm{I}-, \mathrm{P}-$ and $\mathrm{B}-$ frames is $P_{I}=51 \%, P_{P}=20 \%$, and $P_{B}=29 \%$. In the analyzed test scenarios six links were applied with different characteristics. The link properties (bandwidth and loss ratio) used in the simulations are given in the next table.

\begin{tabular}{|c|c|c|}
\hline \hline Link no. & Bandwidth $[\mathrm{kbps}]$ & $\begin{array}{c}\text { Packet loss } \\
\text { ratio }\end{array}$ \\
\hline 1 & 100 & 0.0 \\
\hline 2 & 80 & 0.001 \\
\hline 3 & 80 & 0.005 \\
\hline 4 & 60 & 0.01 \\
\hline 5 & 60 & 0.01 \\
\hline 6 & 20 & 0.05 \\
\hline
\end{tabular}

TABLE III: LINK PROPERTIES

The first point of our performance examinations was to analyze the link utilization ratio. We have examined how the proposed interface selection method utilizes the available resources. In our method a heap up buffer is used to collect a definite number of frames (causing a definite delay). When more data are collected in the heap up buffer, the packet distributor can be more effective, because it can find frames with small sizes, which can still fit to the nearly full interface buffer. In the first test scenario we have compared the link utilization of the proposed method with different heap up buffer sizes. The smallest examined buffer size was able to store
20 frames, which is equal to 0.8 seconds delay, while the biggest heap up buffer had 160 frames $\left(d_{B}=6.4 \mathrm{~s}\right)$.

The results show that the higher the heap up buffer, the higher the usage of most reliable channels, while the load of less reliable ones is lower compared to other heap up buffer settings. For example, when the packets are distributed from a 160 frame sized heap up buffer, the worst link with number 6 is even not used, because the packet distributor could find free gaps in the interface buffers more efficiently and fill the reliable link buffers. Some of the links are barely used, while on the best links, signed with number 1 to 3 (Table III.), the entire bandwidth is almostly used. The utilization level of the links varied in time because the heap up buffer level was also varying. The heap up buffer load is determined by the number of frames (or delay) and not by the data size, therefore the data amount measured in bytes can also change in time. Low level of utilization was measured on links with number 4-6 (Table III.). This is due to the average calculations, because the worst links are not used when the actual heap up buffer level is low. Similarly, if the current heap up buffer size is high, all the links are used.

After the link load measurements, the MPEG frame type distribution in the interface buffers was analyzed. The performance of the proposed contentaware interface selection scheme depends on the video frame distribution on the links with different characteristics. Our aim is to deliver the I-frame data packets on the most reliable channels and transmit the $\mathrm{P}-$ and $\mathrm{B}-$-frames on the reliable links if the link capacity is sufficient. Figure 5: illustrates the different frame type distributions in the interface buffers. In the analyzed scenario the heap up buffer size was set to 80 frames, which is equal to $d_{B}=3.2 \mathrm{~s}$ heap up delay. The interfaces are numbered and ordered based on the packet loss ratios, so the interface that uses the most reliable link has number 1 , while the link with the highest drop probability has number 6 . The video bitrate is $300 \mathrm{kbps}$ and the 
I-frame data ratio of the MPEG-4 video stream is $P_{\Gamma}=51 \%$, hence the I-frame data bandwidth requirement is about $150 \mathrm{kbps}$.

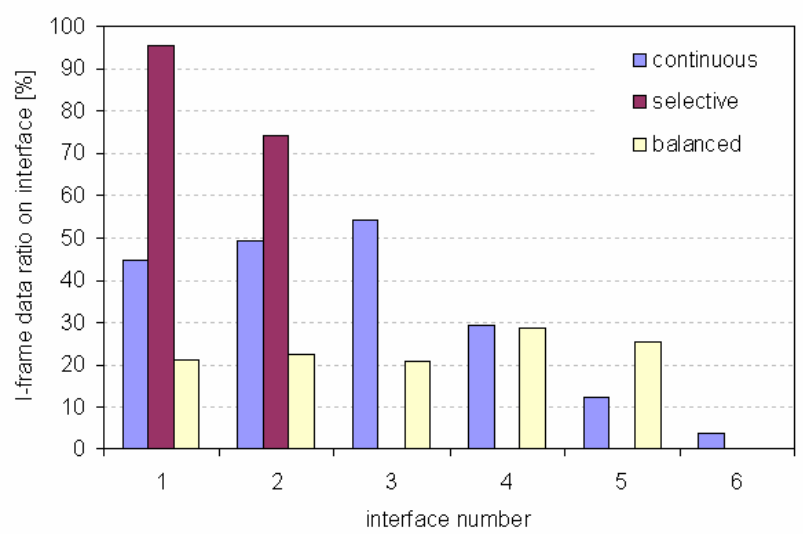

a) I-frame distribution on the interfaces

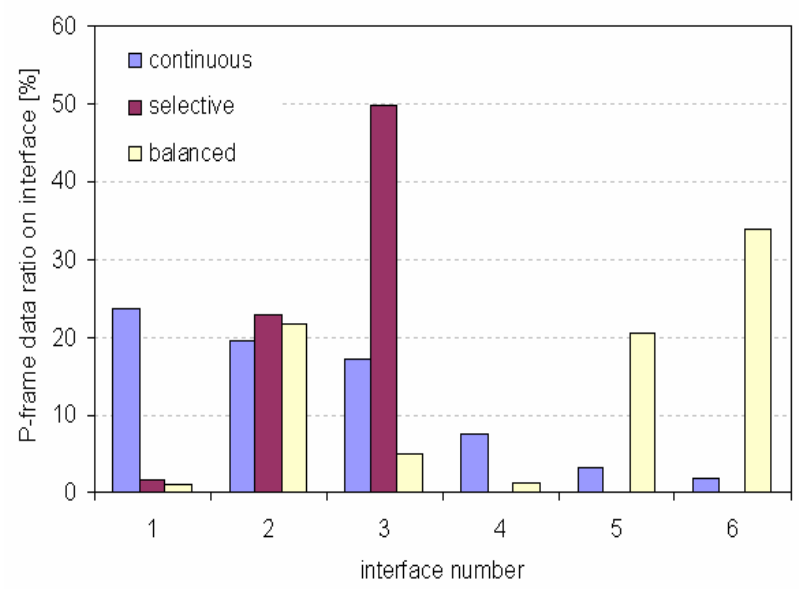

b) P-frame distribution on the interfaces

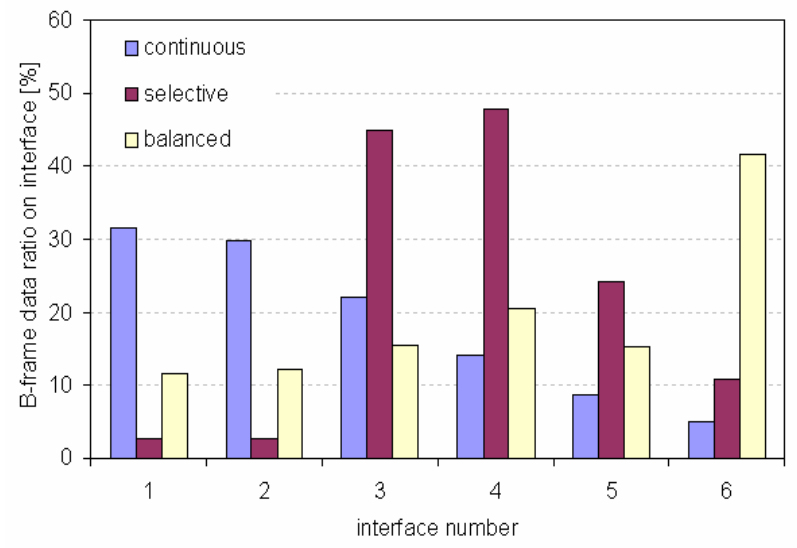

c) B-frame distribution on the interfaces

Figure 5: Different frame type distributions in the interface buffers
As the results show, I-frame data can be delivered on the first two interfaces $(100 \mathrm{kbps}+80 \mathrm{kbps})$ when the proposed content-based selective packet distributor algorithm is used (Figure 5. a). Most of the $\mathrm{P}$-frames were delivered on the $3^{\text {th }}$ interface, while the B-frames were transferred on the less reliable channels. Due to this ordered packet delivery, the number of lost packets belonging to Iframes is estimated to be significantly lower then the number of other frame packet losses.

The observed video quality highly depends on the number of lost packets and the packet content. In order to analyze the behavior of the proposed multipath delivery method we have examined the packet loss of different frame types. The overall packet loss ratio was also measured, which is the average of the I-, $\mathrm{P}-$ and $\mathrm{B}$-frame packet loss ratios. The results are presented in 0Similarly to the previous measurements the heap up buffer size was set to 80 frames.

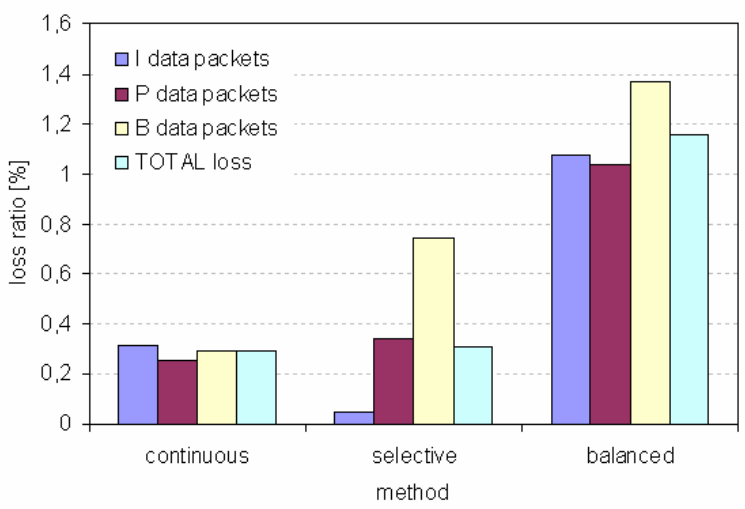

Figure 6: Total and I-, $P-, B$-frame type data packet loss ratios

As we expected, the total number of packet losses was equal using the continuous packet distributor method and the selective one. In spite of the similarity, the number of I-frame data packet losses was significantly lower when the proposed interface selection algorithm was used. In case of the continuous algorithm, the loss probabilities of different types of frame data were nearly identical. However, by protecting the I-frame data, the error 
propagation can be prevented, hence video quality improvement can be achieved. The worst method is the balanced one as shown in 0 , because in this case the packet distributor equally fills the buffers belonging to reliable and unreliable channels.

The main goal of our proposal is to improve the quality of video streams. To see the effectiveness of our scheme we measured the average peak signalto-noise ratio (PSNR) of the transmitted video stream with different heap up buffer size setups. The PSNR values were measured against the original frames applying the content-aware interface selection scheme, the continuous and the balanced method. As it was expected, the measured PSNR value dropped dramatically when the packet loss increased.

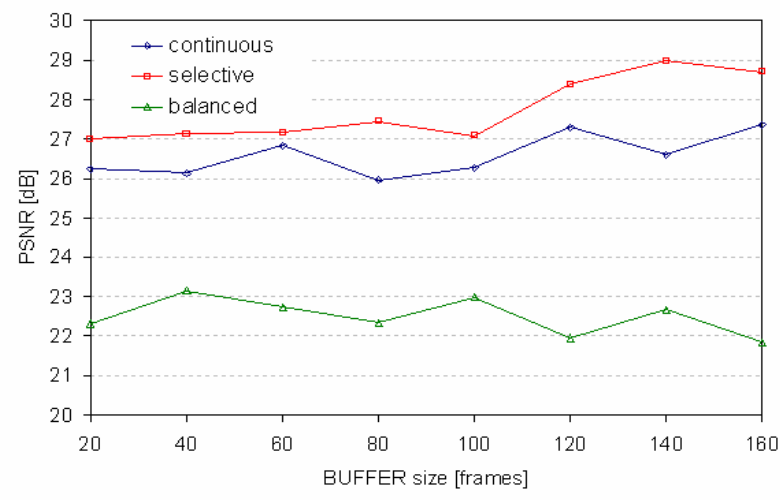

Figure 7: Average PSNR measured with different heap up buffer size setups

The video quality difference between the selective and continuous scheme was not so significant because the total packet loss ratio in case of the two schemes was similar. The performance of the balanced method is very poor. When only the link loss probabilities were taken into consideration (continuous method) the video quality was acceptable. When the packets are distributed based on its content, further quality improvement was achieved. The difference was about $1 \mathrm{~dB}$ in our tests, but of course it can vary significantly according to the link properties and the coded video sequence.

\section{B. Modified GRA-based network selection method}

In Section IV three different normalization techniques for GRA-based network selection decision were presented. Table IV summarizes the original and the proposed solutions.

In order to analyze the efficiency of different normalization methods from the rank reversal phenomenon point of view, we have implemented a simulator tool. Rank reversal depends on the network parameters, disappearing network and normalization method. To compare the different solutions, we have analyzed the frequency of rank order changes caused by network removals. The highest probability of rank reversal can be reached if the worst network is removed, since the disappearance of the worst network is the most realistic.

\begin{tabular}{|c|c|c|c|c|}
\hline & original & norm_1 & norm_2 & norm_3 \\
\hline $\begin{array}{c}\text { smaller- } \\
\text { the- } \\
\text { better }\end{array}$ & $\frac{u_{j}-s_{i}(j)}{u_{j}-l_{j}}$ & $\frac{E_{\max j}-s_{i}(j)}{E_{\max j}-E_{\min j}}$ & $\frac{E_{\max j}-s_{i}(j)}{E_{\max j}-l_{j}}$ & $\frac{l_{j}}{s_{i}(j)}$ \\
\hline $\begin{array}{c}\text { larger- } \\
\text { the- } \\
\text { better }\end{array}$ & $\frac{s_{i}(j)-l_{j}}{u_{j}-l_{j}}$ & $\frac{s_{i}(j)-E_{\min j}}{E_{\max _{j}}-E_{\min j}}$ & $\frac{s_{i}(j)-E_{\min j}}{u_{j}-E_{\min j}}$ & $\frac{s_{i}(j)}{u_{j}}$ \\
\hline
\end{tabular}

TABLE IV: NORMALIZATION TECHNIQUES FOR GRABASED NETWORK SELECTION

In our simulator tool we used random variables as network parameters. According to the defined steps of the GRA method, we have normalized the network parameters (original, norm_1, norm_2, norm_3) and calculated the GRC (Grey Relational Coefficient). As next step we removed the worst network (network with the lowest GRC) and started the GRA process from the beginning. As last step we checked whether the selected network before and after the removal was the same.

We have iterated the described simulation method $10^{6}$ times in order to have statistically correct results. The ratio of total number of iterations and the number of different decisions was calculated, which can be 
considered as the probability of rank reversal. 0shows the obtained results.

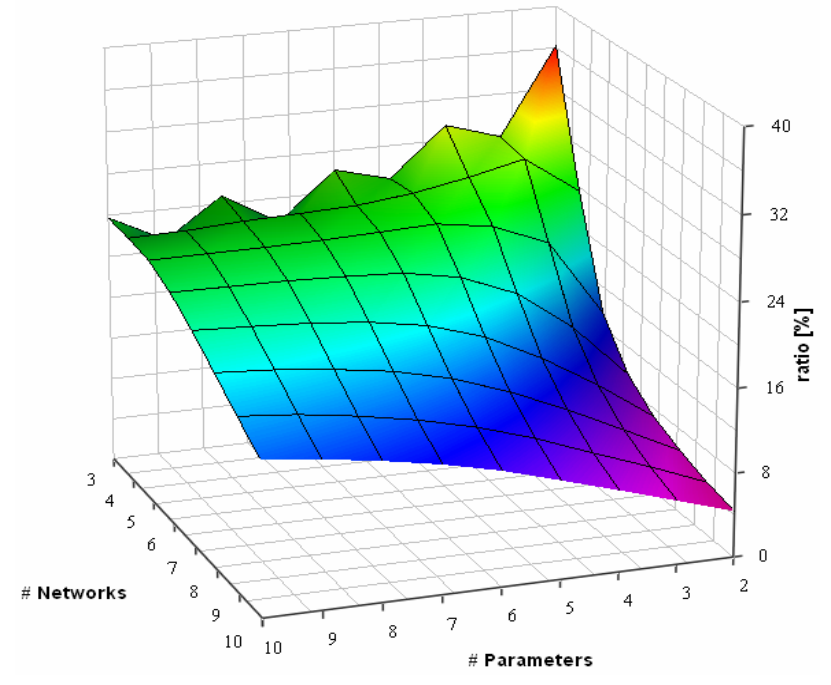

a) original GRA-based network selection decision

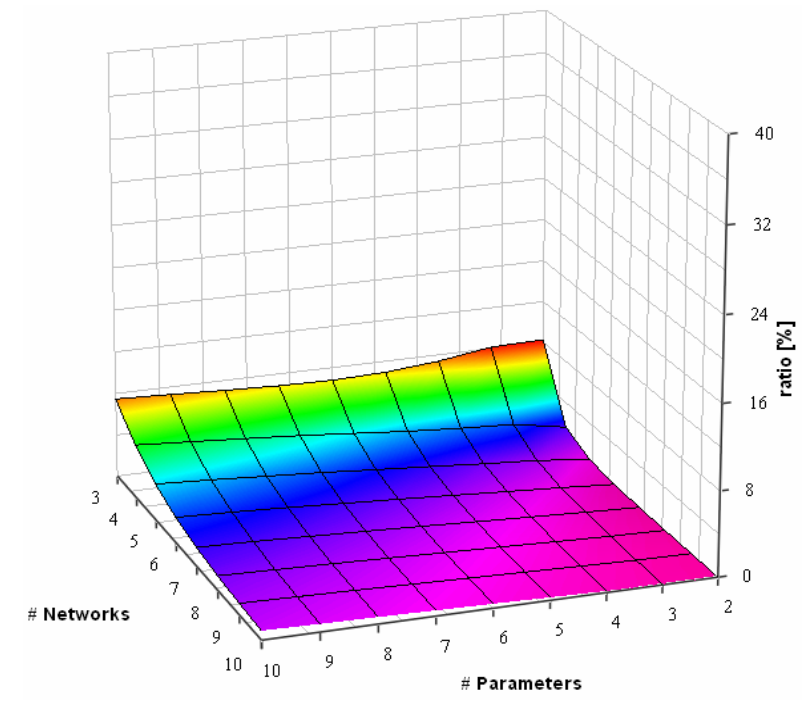

b) of GRA-based network selection decision with norm_2 normalization technique

Figure 8: Rank reversal probability

The number of networks in the simulation was 3 to 10 , while the number of network parameters was 5 . As the results show, the original GRA performance was very bad. In the case when 5 or less networks were reachable and the worst network was removed, the probability of rank reversal was about $25 \%$. Compared to the original GRA method, our techniques (norm_2, norm_3) reduced the rank abnormality by $65 \%$ to $99.9 \%$ as shown in 0 . Using normalization method with absolute min-max values (norm_1), the rank reversal phenomenon was eliminated. With norm_2 and norm_3, the probability that the best network will change after the worst network removal is significantly lower. The performance of norm_2 is very similar to that of norm_3 technique.

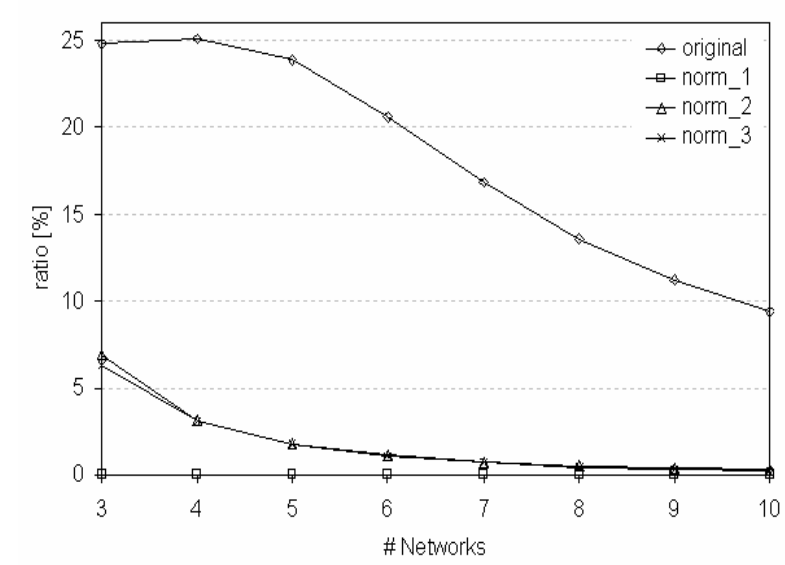

Figure 9: Measured rank reversal probability in case of 5 network attributes

As it can be observed, the number of accessible networks has impact on the rank reversal probability. By increasing the number of available networks the analyzed ratio decreases, because the probability that the maximal $\left(u_{j}\right)$ and minimal $\left(l_{j}\right)$ values will change is lower when the worst network is removed.

We have analyzed the relation between the number of parameters and the rank reversal probability. In the simulations we changed the number of attributes from 2 to 10 . The results of our simulations are presented in OIn 0 a) the efficiency of the original GRA method is presented. As can be seen, in the worst cases the rank reversal probability can reach even $40 \%$. Using norm_2 and norm_3 techniques the rank reversal ratio is decreased. $0 \mathrm{~b}$ ) depicts the performance of norm_ 2 . The performance of norm_3 technique is almost the same.

The impact of the number of network attributes is also observable. By increasing the number of network attributes used for decision making, the rank reversal 
probability increases. When there are more parameters that must be taken into account, the possibility that the removed network has highest or lowest attributes, will be higher.

The results confirm that by using absolute minimal and maximal values defined before for network selection, we can significantly decrease or even eliminate the possibility of the rank reversal phenomenon.

\section{CONCLUSIONS}

The increase of multimedia applications over the Internet has placed new requirements on current video streaming solutions. High bandwidth video provision in mobile environment can also be available if the mobile terminal is equipped with more interfaces. With the effective utilization of the various links, the quality of the video stream can be significantly increased. In this paper a content-aware interface selection scheme was presented, which provides efficient multipath delivery of MPEG video streams. In our solution a heap up buffer is applied to increase the efficiency of the packet distributions transferring the high priority data on the best links. In order to determine the rank order of the links, we have investigated a modified GRA network ordering technique. Our aim was to reduce and eliminate ranking inconsistency, because it can potentially decrease the quality of the link by causing handovers whenever the list of the available networks changes. To evaluate our proposals, simulations were performed and the obtained results proved the efficiency of our multipath transmission scheme combined with modified GRA network ordering solution. Due to comprehensive modeling of video streams, the proposed low complexity solution provides an interesting and effective solution for video streaming in multipath infrastructures.

\section{ACKNOWLEDGMENT}

This work was supported by the Mobile Innovation Centre (MIK) and the OPTIMIX project which is partly funded by the 7th Framework Programme of the European Commission's Information Society Technology.

\section{REFERENCES}

[1] J. L. Deng, "Introduction to grey system theory", The Journal of Grey System, vol.1, no. 1, pp. 1-24, 1989.

[2] Q. Song and A. Jamalipour, "A network selection mechanism for next generation networks", IEEE ICC, pp. 1418--1422, May 2005.

[3] Dimitris Charilas, Ourania Markaki, Dimitris Nikitopoulos, Michael E. Theologou, "Packet-switched network selection with the highest QoS in $4 G$ networks", Elsevier, Computer Networks 52(1): 248-258 (2008)

[4] Gy. Godor, G. Detari, "Novel Network Selection Algorithm for Various Wireless Network Interfaces", Mobile and Wireless Communications Summit, 2007. 16th IST, 1-5 July 2007

[5] Yiyo Kuoa, Taho Yang, Guan-Wei Huang, "The use of grey relational analysis in solving multiple attribute decision-making problems", Elsevier, Computers \& Industrial Engineering, Volume 55, Issue 1, pp. 80-93, August 2008

[6] L. Golubchik, J. Lui, T. Tung, A. Chow, and W. Lee, "Multi-path continuous media streaming: What are the benefits?," ACM J. Perform. Eval., vol. 49, no. 1-4, pp. 429-449, Sep. 2002.

[7] J. G. Apostolopoulos and M. D. Trott, "Path diversity for enhanced media streaming," IEEE Commun. Mag., vol. 42, no. 8, pp. 80-87, Aug. 2004.

[8] Pascal Frossard, Juan Carlos de Martin, Reha Civanlar, "Media Streaming with Network Diversity", Invited paper, Proceedings of the IEEE, vol. 96, no 1, pp. 39-53, January 2008.

[9] Y. J. Liang, E. Setton, B. Girod, "Channel-adaptive video streaming using packet path diversity and ratedistortion optimized reference picture selection," Workshop on Multimedia Signal Processing, 2002.

[10] S. Lin, S. Mao, Y. Wang, and S. Panwar, "A reference picture selection scheme for video transmission over adhoc networks using multiple paths," in IEEE ICME, 2001.

[11] R. Karrer, T. Gross, "Multipath streaming in best-effort networks", ICC 2003, Anchorage, Alaska, May 2003 
[12] Jacob Chakareski, Pascal Frossard, "Distributed Collaboration for Enhanced Sender-Driven Video Streaming”, IEEE Transactions on Multimedia, vol. 10, no 5, pp. 858-870, August 2008.

[13] Ahsan Habib, John Chuang, "Multihoming Media Streaming”, IEEE Workshop on Multimedia Systems and Networking, WMSN 2005, April 2005.

[14] A. C. Begen, Y. Altunbasak, O. Ergun, and M. M. Ammar, "Multi-path selection for multiple description encoded video streaming”, EURASIP Signal Processing: Image Comm., vol. 20, pp. 39-60, Jan. 2005.

[15] Dan Jurca, Pascal Frossard, "Video Packet Selection and Scheduling for Multipath Video Streaming”, IEEE Transactions on Multimedia, vol. 9, no. 3, pp. 629-641, April 2007.

[16] R. Stewart (Ed.): Stream Control Transmission Protocol, IETF RFC 4960, September 2007.

[17] G. Ye, T.N. Saadawi and M. Lee: "IPCC-SCTP: an enhancement to. the standard SCTP to support multihoming efficiently," IPCCC 2004, Phoenix, Arizona, April 15-17, 2004

[18] J. Iyengar, P. Amer, R. Stewart, "Concurrent multipath transfer using SCTP multihoming over independent endto-end paths", IEEE/ACM Trans on Networking, 12/06.

[19] N.D. Tripathi, J.H. Reed and H.F. Vanlandinghum, "Adaptive handoff algorithm for cellular overlay systems using fuzzy logic”, IEEE Vehicular Technology Conference, pp. 1413-1418, 16-20 May 1999.

[20] Y. Gwon, D. Funato, and A. Takeshita, "Adaptive approach for locally optimized IP handoffs across heterogeneous wireless networks," MWCN '02, pp. 475-479, Stockholm, Sweden, September 2002.

[21] M. Ylianttila, M. Pande, J. Mäkelä, and P. Mähönen, "Optimization scheme for mobile users performing vertical handoffs between IEEE 802.11 and GPRS/EDGE networks," in Proceedings of the IEEE Global Telecommunications Conference (GLOBECOM '01), vol. 6, pp. 3439-3443, San Antonio, Tex, USA, November 2001.

[22] H. S. Park, S. H. Yoon, T. H. Kim, J. S. Park, M. S. Do, and J. Y. Lee, "Vertical handoff procedure and algorithm between IEEE802.11 WLAN and CDMA cellular network, " CIC '02, Seoul, Korea, October 2002

[23] C.L. Hwang, K. Yoon, Multiple Attribute Decision Making: Methods and Applications, Springer-Verlag, Berlin, 1981.
[24] M. Zeleny, "Multiple Criteria Decision Making”, McGraw-Hill, 1982.

[25] K. Yoon, C.L. Hwang, "Multiple Attribute Decision Making Introduction”, ISBN: 978-0803954861, Sage Publication, 1995

[26] Eduardo Parente Ribeiro and Victor C. M. Leung, "Minimum Delay Path Selection in Multi-Homed Systems with Path Asymmetry", IEEE Communications Letters, Vol. 10, No. 3, March 2006

[27] F. Bari and V. Leung, "Multi-attribute network selection by iterative TOPSIS for heterogeneous wireless access", in Proceedings of the 4th IEEE CCNC '07, pp. 808-812, Las Vegas, Nev, USA, January 2007.

[28] J. Ylitalo, T. Jokikyyny, T. Kauppinen, A. J. Tuominen, and J. Laine, "Dynamic network interface selection in mobile hosts," in Proceedings of the 36th Annual Hawaii International Conference on System Sciences (HICSS '03), pp. 315-324, Big Island, Hawaii, USA, January 2003

[29] F. Bari and V. Leung, "Application of ELECTRE to network selection in a hetereogeneous wireless network environment," in Proceedings of the IEEE WCNC '07, pp. 3810-3815, Kowloon, Hong Kong, March 2007.

[30] A. Roveri, C. Chiasserini, M. Femminella, T. Melodia, G. Morabito, M.Rossi, and I. Tinnirello, "The RAMON Module: Architecture Framework and Performance Results",

[31] Y-M Wang, T. M.S. Elhag, "An approach to avoiding rank reversal in AHP”, Elsevier, Decision Support Systems (2006) pp. 1474-1480

[32] J. Barzilai, B. Golany, "AHP rank reversal, normalization and aggregation rules", INFOR vol. 32., num. 2, pp. 57-63., 1994

[33] T. D. Neame, M. Zukerman, R. G. Addie, “Application of the M/Pareto Process to Modeling Broadband Traffic Streams", IEEE ICON '99, Brisbane, Australia, 1999.

[34] T. D. Neame, M. Zukerman, R. G. Addie, "Applying Multiplexing Characterization to VBR Video Traffic", ITC 1999 16, June 1999.

[35] FFmpeg Project, http://ffmpeg.mplayerhq.hu/. 


\section{AUTHOR BIOGRAPHIES}

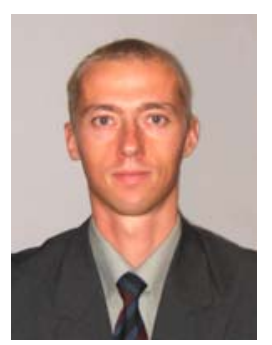

Árpád Huszák graduated with M.Sc. degree in Electronic Engineering from the Budapest University of Technology and Economics (BUTE) at the Department of

Telecommunications, in 2003. He is currently a $\mathrm{PhD}$ candidate at the same university, member of Mobile Communications and Computing Laboratory and student member of IEEE. His research interests focus on network protocols, mobile computing and adaptive multimedia communication over wireless networks.

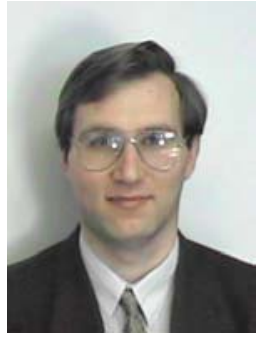

Sándor Imre was born in Budapest in 1969. He received the M.Sc. degree in Electronic Engineering from the Budapest University of Technology and Economics (BUTE) in 1993. Next he started his $\mathrm{Ph}$. D. studies at BUTE and obtained dr. univ. degree in 1996, Ph.D. degree in 1999 and DSc degree in 2007. Currently he is carrying his teaching activities as Head of the Dept. of Telecommunications of BUTE. He was invited to join the Mobile Innovation Centre of BUTE as R\&D director in 2005. His research interest includes mobile and wireless systems. Especially he has contributions on different wireless access technologies, mobility protocols and reconfigurable systems. 